\title{
Los Humedales de la Quebrada Estero en San Ramón, Costa Rica: su importancia y estado actual
}

\author{
Wetlands of Estero Creek in San Ramón, Costa Rica: \\ importance and current status
}

\author{
Cindy Elena Rodríguez-Arias ${ }^{1}$ \\ Ana Margarita Silva Benavides ${ }^{2}$ \\ Universidad de Costa Rica, Costa Rica
}

\begin{abstract}
Resumen. Los humedales de la microcuenca alta de la Quebrada Estero, en San Ramón, Costa Rica, son considerados una prioridad de conservación por su representatividad y ubicación que los hace únicos en su tipo. Por eso se planteó el objetivo de conocer su importancia y estado actual para que esta información sirva de base para su recuperación y conservación. Se llevó a cabo la identificación de los servicios ecosistémicos y de las amenazas e impactos que tienen las actividades humanas en ellos mediante visitas de campo, análisis de uso del suelo, revisión de información secundaria y consulta a actores clave. Los servicios ecosistémicos son el reabastecimiento de aguas subterráneas, depuración del agua, control biológico, control de inundaciones, protección de suelos y sedimentos, adaptación al cambio climático, mantenimiento de la cadena trófica, hábitat para la flora y fauna, provisión de agua para actividades agrícolas y domésticas, alimento para ganado, recursos genéticos y son un elemento importante del patrimonio cultural y natural de San Ramón. Por otro lado, están amenazados por la rápida urbanización que provoca contaminación del agua por fuentes domésticas, proliferación de especies invasoras, pérdida del hábitat acuático, rellenos, invasión de las zonas de protección, eutrofización y colmatación de los embalses y disminución de su capacidad para mitigar inundaciones. Por lo tanto, debido al estado de degradación y ante la falta de un ecosistema de referencia o información ecológica previa a la degradación, se recomienda llevar a cabo acciones de rehabilitación ecológica para recuperar y conservar los servicios ecosistémicos que brindan.
\end{abstract}

Palabras clave. Humedales, servicios ecosistémicos, amenazas, rehabilitación ecológica

\begin{abstract}
The high micro-basin wetlands of the Estero Creek in San Ramon, Costa Rica are considered a conservation priority for their representativeness and location, which makes them unique. The objective of this study was to know their importance and current status so this information serves as a basis for their restoration and conservation. The identification of ecosystem services and the threats and impacts of human activities on them was carried out through field visits, analysis of land use, review of secondary information, and consultation with key actors. The ecosystem services are replenishing groundwater, water purification, biological control, flood control, protection of soil and sediment, adaptation to climate change, sustaining food chains, habitat for flora and fauna; water supply for agricultural and domestic activities, livestock feed, genetic resources, and they are an important part of the cultural and natural heritage of San Ramon. On the other hand, they are threatened by rapid urban development, which causes water pollution from domestic sources, spread of invasive species, loss of aquatic habitat, landfills, invasion of protected areas, eutrophication and silting of reservoirs, and decrease of their ability to mitigate floods. Therefore, due to the state of degradation and the lack of a reference ecosystem or ecological information prior to degradation, ecological rehabilitation actions to restore and preserve the ecological services the wetlands provide are recommended.
\end{abstract}

Keywords. Wetlands, ecological services, threats, ecological rehabilitation

${ }^{1}$ Cindy Elena Rodríguez-Arias, Sección de Biología, Sede de Occidente de la Universidad de Costa Rica. Dirección electrónica: cindy.rodriguez_a@ucr.ac.cr ${ }^{2}$ Ana Margarita Silva Benavides, Escuela de Biología, Centro de Investigación en Ciencias del Mar y Limnología (CIMAR), Universidad de Costa Rica Dirección electrónica: ana.silva@ucr.ac.cr 


\section{Introducción}

Los humedales son zonas que están temporal o permanentemente inundadas, son altamente productivos y constituyen un componente vital del ciclo hidrológico (Anda-Martín, et al., 2013). Además, ofrecen una serie de bienes y servicios ecosistémicos de gran valor (Secretaría de la Convención de Ramsar, 2013). A pesar de eso, están desapareciendo rápidamente en todo el mundo. En particular, los humedales urbanos son los más amenazados por ubicarse dentro de áreas densamente pobladas o en expansión, que los exponen con mayor frecuencia a alteraciones que los degradan y los hacen desaparecer de manera más acelerada que otros ecosistemas (Fox, 2013).

Al oeste de la ciudad de San Ramón, existe un sistema de humedales que forman parte de la microcuenca alta de la Quebrada Estero y que en conjunto se conocen como "Lagunas Quebrada Estero". Dicho sistema está compuesto por la quebrada que les da su nombre y un afluente, dos embalses artificiales conocidos como El Laguito y El Porvenir, creados para evitar inundaciones (Mora, 2007) pero que se ubican en sitios que históricamente fueron humedales y un pantano natural que es remanente de un lago que existió en la zona durante el periodo Cuaternario (Bergoeing y Murillo, 2012),

Este sistema de humedales fue identificado como una prioridad de conservación por su representatividad y ubicación según el análisis de vacíos de conservación en Costa Rica (Sistema Nacional de Áreas de Conservación SINAC, 2007). Esto significa que deben ser conservados como zonas núcleo que orienten la gestión de la biodiversidad dulceacuícola general o funcional a escala de paisaje en la cuenca del Río Grande de Tárcoles (Paaby-Hansen, 2008).

Sin embargo, actualmente presentan problemas de contaminación y degradación producto de la actividad urbana en sus alrededores (Quesada, 2003; Mora, 2007). Debido a esto es necesario conocer el estado de conservación actual de estos ecosistemas, su funcionalidad básica, los servicios ecosistémicos provistos a las comunidades locales (Gallo y Rodríguez, 2010) y las causas que han llevado a su degradación, para poder proponer un proceso eficaz de planificación del manejo y el monitoreo (Secretaría de la Convención de Ramsar SCR, 2010a).

El objetivo de esta investigación fue estudiar el estado actual de estos humedales y su importancia para las comunidades naturales y humanas, y que esta información sirva de línea base para el diseño de estrategias para su recuperación y conservación.

\section{Metodología}

Área de estudio: La microcuenca de la Quebrada Estero (figura 1) se ubica en la provincia de Alajuela, cantón de San Ramón, distrito de Alfaro. Forma parte de la subcuenca del río Grande de San Ramón y a la cuenca del río Tárcoles (Paaby-Hansen, 2008).

La parte alta de la microcuenca tiene un área de 249 ha (Mora, 2007) y la zona de vida es la de Bosque Premontano Húmedo. El patrón de lluvias es el característico del Valle Central, la época seca se extiende de enero a abril y la lluviosa de mayo a noviembre (Leandro, Coto y Salgado, 2010). El uso del suelo incluye parches de bosque, cultivos (principalmente café), pastos y residencial, este último uso es el de mayor aumento en las últimas décadas (Mora, 2007). En la microcuenca no existen industrias ni beneficios de café. Además, es importante resaltar que se brinda el servicio de recolección de basura por parte de la Municipalidad, pero no hay alcantarillado sanitario.

Los humedales que se ubican en esta microcuenca son la Quebrada Estero y un afluente sin nombre, un pantano y dos embalses conocidos 


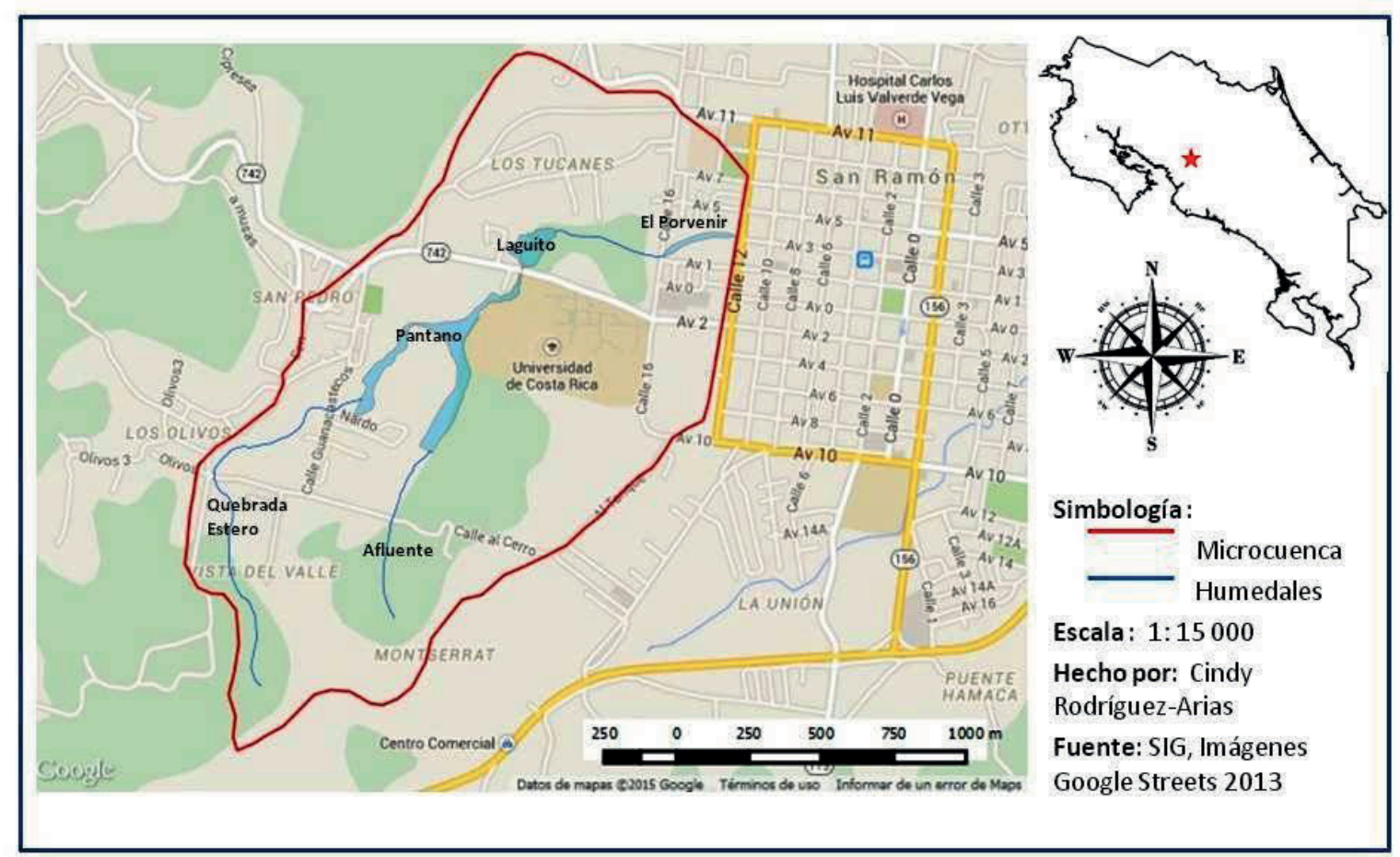

Figura 1. Ubicación de la microcuenca alta de Quebrada Estero y de los puntos de muestreo. Fuente: elaboración propia

como el Laguito y El Porvenir; todos están conectados entre sí por el flujo de agua de la quebrada (figura 1), pero separados por carreteras y alcantarillas (Rodríguez y Silva, 2015).

Se realizaron las siguientes actividades: (a) revisión de información secundaria, (b) visitas a los humedales (una vez al mes entre marzo del 2014 y febrero del 2015 y cada tres meses hasta octubre del 2016) y (c) consulta a actores clave como funcionarios del Ministerio de Ambiente y Energía, de la Universidad de Costa Rica, de la Municipalidad y vecinos de la microcuenca.

Para identificar los servicios ecosistémicos de los humedales se utilizó como guía la lista de chequeo de la SCR (2010a) (anexo 1). Por otro lado, para identificar las principales amenazas provocadas por las actividades humanas a los ecosistemas acuáticos se utilizó la lista de chequeo del Instituto de Estudios Ambientales IDEA (2008) (anexo 2).
Se realizó un análisis de cambio de uso del suelo con el programa Quantum Gis 2.8.1 con base en la fotografía aérea de Google Satellite del 21 de diciembre del 2013 y complementada con verificación de campo. El resultado se comparó con el elaborado por Mora (2007) en el año 2005.

\section{Resultados y discusión}

El concepto de servicios ambientales o ecosistémicos permite establecer una relación directa o indirecta entre el estado y el funcionamiento de los ecosistemas con el bienestar humano (Alcocer, Barba, Díaz y Jiménez, 2016). Según Acosta, Alvarez y colaboradores (2016), estos servicios se clasifican en tres tipos: (a) provisión, (b) regulación y (c) cultural. Es importante que la comunidad conozca de estos servicios, pues las decisiones que las sociedades toman en torno al manejo de los ecosistemas tienen consecuencias en la provisión y en la gestión de los servicios ecosistémicos (Acosta, Alvarez, et al., 2016; Amsteins, 2013). 
De acuerdo con la información obtenida, se identificaron los siguientes servicios ambientales provistos por los humedales de la Quebrada Estero:

Servicios ambientales de regulación. Son consecuencia de los procesos complejos que mantienen las condiciones del ambiente donde las poblaciones humanas habitan y desarrollan sus actividades productivas.

- Constituyen un reservorio de agua dulce y permiten el reabastecimiento de aguas subterráneas, ya que se ubican sobre el acuífero San Ramón-Palmares.

- Purificación de aguas por medio de la retención de contaminantes, depuración o dilución de aguas residuales que se vierten a la quebrada (Rodríguez y Silva, 2015).

- El área del pantano y los embalses son un importante medio de control de inundaciones, ya que retienen grandes cantidades de agua durante las tormentas, lo que permite la disminución de la intensidad de los efectos de las inundaciones en la ciudad de San Ramón (Mora, 2007).

- Contribuyen a la protección de suelos, sedimentos y retención de nutrientes.

- Importantes para la adaptación al cambio climático, particularmente para la amortiguación de inundaciones provocadas por condiciones climáticas extremas que serán más frecuentes en el futuro.

- Existen agentes de control biológico para plagas que transmiten enfermedades, por ejemplo, peces y anfibios que controlan las poblaciones de larvas de zancudos (M. Cartín, comunicación personal, 11 de octubre, 2016; M. Zumbado, comunicación personal, 11 de octubre, 2016).

- Oferta de hábitats y alimento para fauna silvestre acuática y terrestre (Guido y Rodríguez, 2011; M. Cartín, comunicación personal, 11 de octubre, 2016; M. Zumbado, comunicación per- sonal, 11 de octubre, 2016). Son de especial importancia para las especies acuáticas ya que son los ecosistemas que más deterioro han presentado en la región (Guido y Rodríguez, 2011).

- Presencia de poblaciones de flora y fauna silvestre de importancia para la conservación, por ejemplo, las aves acuáticas migratorias (Guido y Rodríguez, 2011), plantas acuáticas (Rodríguez y Silva, 2016) y una especie endémica de pez (M. Zumbado, comunicación personal, 11 de octubre, 2016).

Servicios ambientales de aprovisionamiento. Son los recursos que resultan de la transformación de los ecosistemas realizada por los seres humanos.

- Algunos vecinos toman el agua de las quebradas para uso en actividades agrícolas y domésticas.

- En un sector del pantano, el humedal provee de alimento para el ganado. El manejo de la vegetación invasora podría utilizarse como forraje o para compostaje.

- Es un importante reservorio de material genético.

Servicios ambientales culturales. Son los beneficios no tangibles que se derivan de las interacciones entre los individuos y los ecosistemas, los cuales se obtienen a través de experiencias o a partir de la adquisición de sentimientos de identidad y de autoestima gracias al bagaje cultural.

- Permiten que se lleven a cabo actividades de estudio de la naturaleza y su relación con el ser humano, por lo que reviste gran importancia para las investigaciones científicas y la educación ambiental para las comunidades vecinas.

- Importante patrimonio cultural y natural para la comunidad de San Ramón, en especial el embalse el Laguito, el cual fue un querido centro turístico y recreacional que le dio a la población un sentido de pertenencia que los motiva a parti- 
cipar en su rehabilitación (Rodríguez, Barrantes, Guido y González, 2012).

- Registro histórico preservado en los sedimentos: según Horn (2007), los núcleos de sedimentos de los lagos y humedales de Costa Rica proporcionan evidencia clave para reconstruir los cambios en el clima y los ambientes del pasado y para comprender la forma en que los humanos han utilizado el paisaje. En marzo de 2014, un equipo de investigadores de la Universidad de Tennessee y la Universidad de Costa Rica obtuvo un núcleo de sedimento de aproximadamente $4 \mathrm{~m}$ de profundidad en el pantano. Preliminarmente, pequeños trozos de carbón extraídos del núcleo de sedimento a una profundidad aproximada de $3 \mathrm{~m}$ fueron sometidos a un análisis de radiocarbono y se determinó que tenían $2527 \pm$ 28 años de antigüedad. Este carbón podría haber sido resultado de fuegos naturales causados por un relámpago, o de los fuegos provocados por pobladores. Análisis adicionales proporcionarán más información sobre el historial de incendios en el sitio y el análisis del polen revelará la historia de la vegetación (Horn, comunicación personal, 18 de setiembre, 2014).

- Potencial valor paisajístico.

A pesar de estos servicios ambientales que brindan estos humedales, los impactos de las acciones antrópicas en sus alrededores amenazan su persistencia en el tiempo. Debido a esto es necesario y urgente evaluar los efectos que las actividades humanas tienen en la estructura y función de los ecosistemas con el fin de mitigar sus consecuencias, redefinir y establecer áreas de conservación y generar programas de restauración o rehabilitación ecológica en las zonas que lo requieran (Acosta, Bastida, et al., 2016). Las principales amenazas detectadas para estos humedales son:

- Fragmentación: la presencia de alcantarillas por donde pasan carreteras y caminos, muros y represas causa la pérdida de conectividad ecológica, lo cual imposibilita el movimiento de algunas especies acuáticas como peces. Esto afecta tanto procesos ecológicos como la dispersión de semillas y depredación y puede resultar en la reducción o interrupción del flujo genético, con la consecuente pérdida de diversidad genética, aumento de la endogamia y alteración de los patrones reproductivos (Acosta, Bastida, et al., 2016).

- Deficiente calidad de agua que ingresa a los humedales: existen descargas de aguas residuales domésticas, e incluso aguas negras, lo que provoca eutrofización y mala calidad de hábitat para flora y fauna (Rodríguez y Silva, 2015).

- Pérdida del área inundable y de la ronda: este problema se presenta en el pantano, en el embalse el Laguito y en el embalse El Porvenir. En el primero se rellenó el humedal para ampliar las zonas de potreros y en los dos últimos se ha perdido el área inundable debido a la eutrofización y acumulación de sedimentos.

- Pérdida de la capacidad para mitigar inundaciones: esto se relaciona con el punto anterior. Ocurre principalmente en los embalses por pérdida de la capacidad de almacenar agua, lo que disminuye su capacidad para atenuar caudales y provoca inundaciones y desbordamientos. Esto se agrava durante los eventos extremos de precipitación que ocurren en el Valle Central de Costa Rica por la influencia de factores naturales como El Niño (ENOS), La Niña, movimientos latitudinales del Centro de Convergencia Intertropical, tormentas tropicales y huracanes, la influencia del Atlántico y los frentes fríos.

- Déficit hídrico: cerca de las nacientes se da la extracción de agua para usos domésticos y agrícolas, lo cual puede afectar el caudal de la quebrada en época seca. Además, el fenómeno El Niño ocasionó que la época seca del 2016 fuera más intensa que lo normal, esto provocó que la Quebrada Estero se secara en su naciente y parte 
alta. El bajo caudal que se mantuvo en la naciente del afluente y el agua que nace directamente en el pantano no fue suficiente y provocó que se secara totalmente el embalse el Laguito durante el mes de abril.

- Depósitos de escombros y basuras: a pesar de que existe el servicio de recolección de basura, se observaron cantidades importantes de desechos sólidos en todos los humedales, lo que afecta la calidad del agua y de los suelos. Esto se debe a que en general los humedales son poco apreciados y hasta considerados como sitios hostiles y peligrosos por ciertos sectores de la población (Marín-Muñiz y Hernández, 2016). Además, el depósito de escombros le va robando terreno a los humedales lo que afecta la estructura espacial del ecosistema y sus componentes característicos (Calderón, 2008).

- Pastoreo de animales: ocurre en una sección del Pantano. Esta actividad puede causar compactación del suelo y aportes de excrementos al cuerpo de agua (IDEA, 2008).

- Presencia de predadores domésticos: por su cercanía a centros urbanos hay presencia de ratas, perros y gatos que pueden atacar la escasa fauna de aves, peces, reptiles y anfibios que aún persiste en la microcuenca, matando tanto a individuos adultos o juveniles como sus huevos. Incluso, la afectación puede ser indirecta, por ejemplo, la simple presencia de los perros en las rondas, como la de cualquier otro depredador, causa perturbaciones en el comportamiento de las aves acuáticas como apareamiento, incubación y alimentación, impidiendo que los adultos se reproduzcan y que los juveniles lleguen a la etapa de madurez. También pueden generar contaminación por sus excrementos y son vectores de muchas enfermedades zoonóticas que se transmiten a la vida silvestre (Calderón, 2008).

- Modificación en la composición y estructura de las comunidades bióticas: puede ocurrir pérdida de especies nativas por proliferación de invasivas y pérdida de especies acuáticas susceptibles a las altas cargas orgánicas del agua y a la baja oxigenación (IDEA, 2008). En el caso de esta microcuenca, algunos sectores del pantano están dominados por la especie Coix lacryma-jobi (Lágrimas de San Pedro), exótica e invasiva (Rodríguez y Silva, 2016). Algunas especies exóticas desplazan a las nativas y pueden incluso llegar a causar su extinción local debido a interacciones como la competencia o la depredación / herbivoría, o por ser portadoras de enfermedades (Acosta, Bastida, et al., 2016).

- Prácticas de manejo inadecuadas o ausentes: en varias ocasiones se evidenció el uso de herbicidas por parte de funcionarios municipales para el control de la vegetación en el embalse El Porvenir. Este método debe ser descartado por sus problemas de aplicación, sus efectos sobre especies distintas de las atacadas (Del Olmo, 2008) por la toxicidad sobre la biota natural de los humedales (Secretaría Distrital del Ambiente SDA, 2008). En los demás puntos no existe ningún tipo de manejo, excepto el pastoreo en algunas partes del pantano.

- Apropiación y uso inadecuado de las áreas de ronda: se han dado construcciones de viviendas y edificios sin respetar las zonas de protección definida por la legislación nacional. Dicha área, donde se debería preservar el bosque natural ribereño, es fundamental para proteger y conservar los humedales, ya que proveen servicios ecosistémicos como protección de la fauna y flora silvestre, regulación del clima, mejora de la conectividad, protección del recurso hídrico, protección y recuperación de los suelos, reducción de la vulnerabilidad, ampliación deespacios verdes, belleza escénica, entre otros (Sánchez, Scorza, Feoli y Artavia, 2015). A pesar de esto, y de que existe un mecanismo legal (La Ley Forestal No.7575) que permite asegurar la protección del cauce, su ejecución no se hace efectiva por 
parte de las autoridades, por tanto se permiten construcciones que invaden estas áreas. En el caso particular de la Quebrada Estero, hay una larga historia de inundaciones debidas a este problema (Quesada, 2003).

- Problemas relacionados a los derechos de propiedad y uso: la legislación costarricense es dispersa y confusa respecto a los humedales ubicados en propiedad privada ya que por un lado, el decreto ejecutivo número 35803 en su Artículo $2^{\circ}$, dice que "Los ecosistemas de humedales continentales forman parte del Patrimonio Natural del Estado", pero por otro lado, la norma citada no es aplicable a los humedales localizados en propiedad privada, porque son terrenos que no están inscritos a nombre del Estado. Además, el artículo 32 de la Ley Orgánica del Ambiente establece que los humedales pueden ser declarados área silvestre protegida bajo la categoría de manejo humedal. Otra confusión es en el sentido de que no siempre un humedal (entendido como ecosistema) constituye Patrimonio Natural del Estado, en los términos descritos (Sistema Nacional de Áreas de Conservación/SINAC- Programa de las Naciones Unidas para el Desarrollo/ PNUD y Proyecto Humedales, 2015). En el caso de los humedales en cuestión, gran parte del recorrido de la quebrada, el afluente y el pantano están en manos privadas lo que limita el acceso a algunos de los puntos y se requiere del permiso y aprobación de los propietarios para cualquier acción que se quiera realizar. Parte del Pantano y el Laguito pertenecen a la UCR donde ya fueron declarados como áreas protegidas y el embalse El Porvenir pertenece a la Municipalidad.

- La ausencia de una valoración ambiental y económica de los servicios ecosistémicos que provee el humedal, desconocimiento de estos por la mayoría de la sociedad: esto facilita el deterioro y la sobreexplotación pues a la hora de tomar decisiones los criterios económicos están por encima de consideraciones ecológicas o éticas (IDEA, 2008). Por ejemplo, en un estudio realizado por estudiantes de la Universidad de Costa Rica, se encontró que el 59 \% de los encuestados ni siquiera sabían de la existencia del Laguito, principalmente los más jóvenes (González, Jiménez, Jiménez y Palma, 2014). Según Marín-Muñiz y Hernández (2016) este desinterés de las nuevas generaciones por los ecosistemas de humedales ocurre al no haber un uso directo o interacción entre los habitantes y el humedal, por lo que no habrá una apropiación de este por parte de los vecinos, ni interés por preservarlo o usarlo apropiadamente. Por ello, resulta prioritario fomentar desde los jóvenes una educación ambiental que integre los servicios ambientales que los ecosistemas de humedales proveen, aunque no hagan usos directo de los ecosistemas.

- La falta de control y vigilancia en los humedales: esto ocurre en todo el país a pesar de que existe un marco legal de protección a los humedales. No se tiene la correcta vigilancia y faltan acciones y políticas concretas y estrictas para salvaguardarlos, ya que ni siquiera los humedales de importancia internacional Ramsar escapan a esta problemática (García, 2013)

- Desarticulación de las actividades de las instituciones pertinentes: por ejemplo esfuerzos aislados e incompletos, escasa protección, ausencia de manejo, entre otros. En varias ocasiones, desde 1992, se han propuesto proyectos conjuntos para el rescate del embalse El Laguito (Rodríguez et al., 2012) pero la desarticulación, la falta de apoyo político, de recursos y de mantenimiento los ha llevado al fracaso. Todo esto se ve agravado por el hecho de que el ordenamiento jurídico costarricense posee normas dispersas para regular los ecosistemas de humedal, lo que causa muchas confusiones a la hora de actuar y asumir responsabilidades (SINAC-PNUD y Programa Humedales, 2015).

- Actividad urbana adyacente: causa problemas de incompatibilidades de uso del suelo, 
impactos por el tráfico (ruido, contaminación atmosférica y alteraciones del patrón de flujo hídrico, entre otros), barreras a la movilidad, inseguridad, contaminación con basuras y vertimientos, etc. Además, el proceso de urbanización generado por el crecimiento de la población ha impermeabilizado gran parte de la microcuenca, lo que repercute en el incremento de la escorrentía y aumento en la verticalidad de las paredes de los cauces que aumentan la posibilidad de que ocurran inundaciones.

- Cambio en el uso del suelo: en esta microcuenca el uso urbano ha ido en aumento durante las últimas décadas y ha disminuido la cobertura de bosque y cultivos permanentes (Quesada, 2000; Marsik y Waylen, 2006; Mora, 2007). En la figura 2 se puede apreciar que en la actualidad el principal tipo de cobertura en esta microcuenca es el urbano o listo para urbanizar, que ocupa un 45,5\% del área total. Le siguen las áreas de pastos con un 28,4\%, los cultivos (principalmente café) con 17,3 y finalmente las áreas de bosque y regeneración natural con un $8,8 \%$. Si se compara el uso del suelo actual con el realizado por Mora (2007) (tabla 1), se puede apreciar que en los últimos diez años el área destinada al desarrollo urbano creció un $20,1 \%$, mientras que esta misma categoría creció en un 12,15 \% desde 1989 hasta 2005 (Mora, 2007). Por otra parte, durante la última década el área de bosque y regeneración natural aumentó un $7,8 \%$ y el de cultivos un 1, $99 \%$, mientras que la superficie dedicada a pastos disminuyó en un $29,8 \%$ y la de ornamentales en $0,11 \%$.

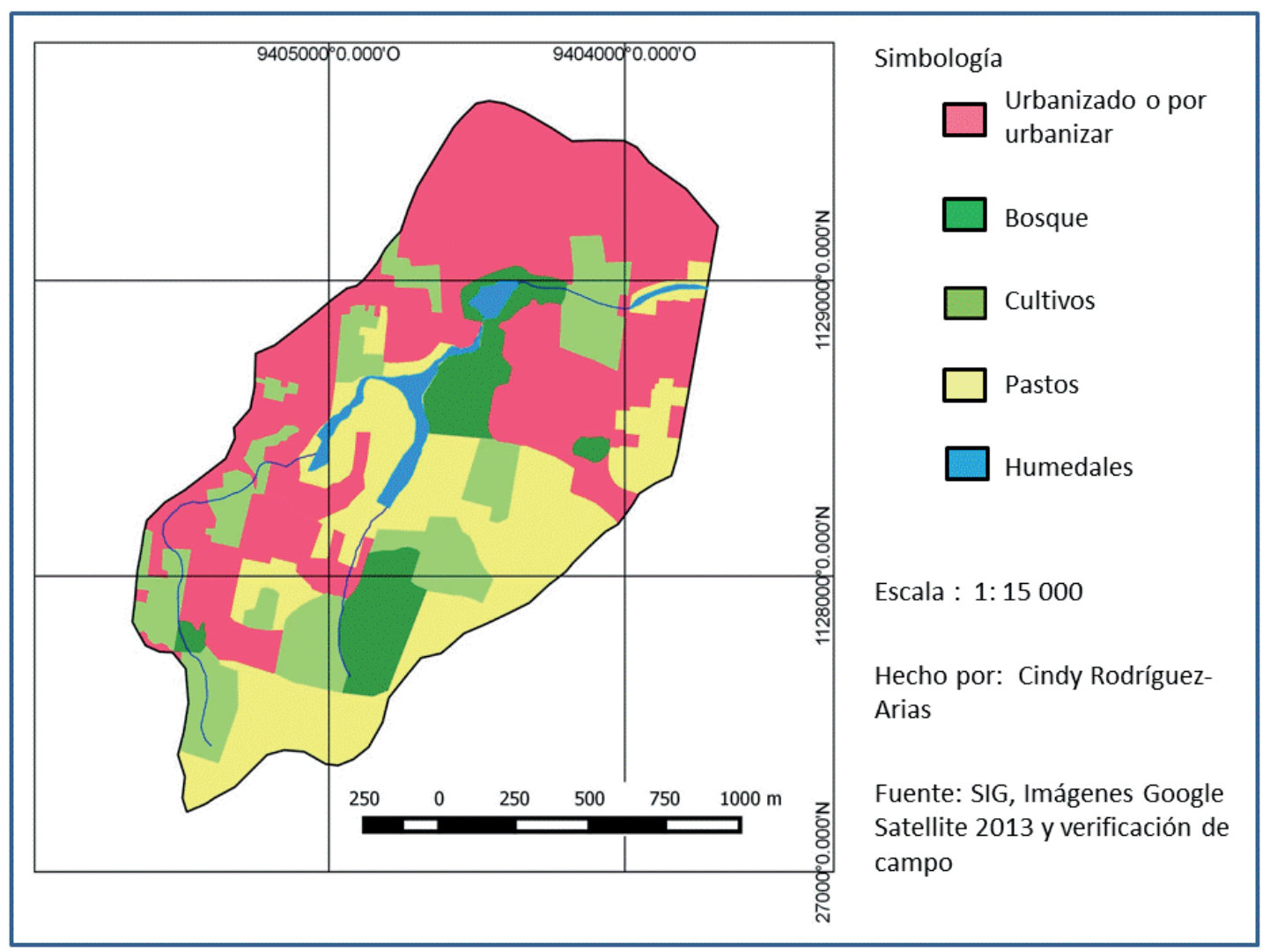

Figura 2. Uso del suelo en la microcuenca alta de la Quebrada Estero en el año 2015. Fuente: elaboración propia. 
Tabla 1

Cambios en el uso del suelo entre 1989 y el 2015

\begin{tabular}{ccc}
\hline & \multicolumn{2}{c}{ Porcentaje del área por tipo de uso } \\
Tipo de uso & 2005 & 2015 \\
\hline Bosque & 0,98 & 8,75 \\
Cultivos & 15,34 & 17,33 \\
Ornamentales y otros & 0,11 & 0 \\
Pastos & 58,15 & 28,40 \\
Urbano & 25,42 & 45,52 \\
\hline
\end{tabular}

Fuente. Quesada, 2001; Mora, 2007; Rodríguez y Silva, 2015.

\section{Conclusiones y recomendaciones}

Los principales servicios ambientales de los humedales de la Quebrada Estero son: el reabastecimiento de aguas subterráneas, depuración del agua, almacenamiento de carbono, control biológico de plagas, control y mitigación de inundaciones, protección de suelos y sedimentos, adaptación al cambio climático global, hábitat y alimento para la flora y la fauna nativa y de interés para la conservación; también proveen agua para algunas actividades agrícolas y domésticas, alimento para ganado, recursos para compostaje y material genético. Finalmente, constituyen un elemento importante del patrimonio cultural y natural de la ciudad de San Ramón, de gran valor paisajístico y que permite hacer actividades de investigación, de educación ambiental, recreativas, culturales, entre otras.

Por otra parte, las principales amenazas a estos ecosistemas incluyen la fragmentación del sistema de humedales, la contaminación del agua, la invasión del área inundable y las rondas, la pérdida de capacidad de mitigar inundaciones debido a la eutrofización y colmatación, depósito de escombros y basura, pastoreo y depredadores domésticos; prácticas de manejo inadecuadas o inexistentes, invasión de especies que provocan cambios en la estructura y composición de las comunidades biológicas, falta de control y vigilancia, crecimiento acelerado de la urbanización en la microcuenca y los cambios en el uso del suelo que esto provoca.

El estado de degradación que experimenta esta microcuenca hace que la restauración ecológica no sea una opción viable pues no existe un ecosistema de referencia ni información sobre estos humedales antes de su degradación que permitan establecer qué se quiere restaurar. Además, los costos de la restauración y su mantenimiento serían muy altos y recurrentes. Debido a esto, se recomienda apelar a estrategias de rehabilitación ecológica que permitan restablecer la provisión de servicios de mitigación de inundaciones, hábitat para la vida silvestre, recreación, educación ambiental e investigación científica.

Entre las acciones recomendadas están: el dragado y posterior mantenimiento de los embalses, protección y reforestación de las nacientes, eliminación de drenajes y rellenos en el pantano, armonizar las obras hidráulicas con las necesidades del ecosistema (pasos de fauna, bahías de inundación, entre otras), aplicar de la normativa vigente sobre control de vertidos a fuentes superficiales de agua, educar a la población sobre buenas prácticas ambientales tanto en el hogar como en las actividades agrícolas, campañas de recolección de basura, rehabilitar la vegetación nativa de las riberas para evitar el arrastre de sedimentos, monitorear y controlar la presencia de especies exóticas, invasoras o depredadoras, 
reintroducción de especies nativas, mejorar la conectividad entre humedales y entre estos y otros elementos del paisaje como los parches de bosque, entre otras.

\section{Agradecimientos}

Este artículo forma parte de la tesis de Cindy Rodríguez en el Programa Doctorado en Ciencias Naturales para el Desarrollo (DOCINADE) de la Universidad Nacional de Costa Rica, el Instituto Tecnológico de Costa Rica y la Universidad Estatal a Distancia, por lo que agradecemos a la Universidad de Costa Rica que por medio de las Becas SEP-CONARE aportó la jornada de la autora Rodríguez. También agradecemos al Ministerio de Ciencia y Tecnología al hacer posible mediante el Programa de Incentivos del CONICIT que se realizara esta investigación. Asimismo, a Marco Zumbado Echavarría quien colaboró con el trabajo de campo.

\section{Referencias}

Acosta, G.; Álvarez, A.; Arellano, L.; Armenta, J.; Caro, Á.; Díaz, V. ... Wong, P. (2016). Los servicios ecosistémicos. En: P. Balvanera, J. Arias-González, R. Rodríguez-Estrella, L. Almeida-Leñero y J. Schmitter-Soto (Ed.), Una mirada al conocimiento de los ecosistemas de México. (pp. 229- 340). México D.F.: Universidad Nacional Autónoma de México.

Acosta, G.; Bastida, R.; Antonio, S.; Cabrera, S.; Castro, J.; Chapa, L.; Chávez, S.; ... Valencia, L. (2016). Impacto de las actividades humanas en la biodiversidad y en los ecosistemas. En: P. Balvanera, J. Arias-González, R. Rodríguez-Estrella, L. Almeida-Leñero y J. Schmitter-Soto (Ed.), Una mirada al conocimiento de los ecosistemas de México. (pp. 57-190). México D.F.: Universidad Nacional Autónoma de México.

Alcocer, J., Barba, E., Díaz, V., Jiménez, M., Lara, A., y Sosa, J. (2016). Cambios en la biodiversidad y sus consecuencias en el fun- cionamiento de los ecosistemas y sus servicios. En: P. Balvanera, J. Arias-González, R. Rodríguez-Estrella, L. Almeida-Leñero y J. Schmitter-Soto (Ed.), Una mirada al conocimiento de los ecosistemas de México. (pp. 191-228). México D.F.: Universidad Nacional Autónoma de México.

Amsteins, K. (2013). Monitoreo ambiental participativo y ciencia ciudadana en el Lago Panguipulli: análisis de caso y propuestas para su conservación, región de Los Ríos, Chile. (Trabajo de Titulación presentado para optar al Título de Ingeniero en Conservación de Recursos Naturales). Universidad Austral de Chile.

Anda-Martín, B., Chavira-Silva, J., Del Toro-Kobzeff, A., Flores-Zavala, R., Jaimes-Lugo, M. y González-Acevedo, Z. (2013). Características ambientales de La Lagunita El Ciprés, Municipio de Ensenada, Baja California y las amenazas a su conservación. Revista GEOS, 33(2), 1-19.

Bergoeing, J. y Murillo, M. (2012). El asentamiento precolombino en San Ramón y su imbricación geomorfológica. Revista Geográfica, 151, 113-128.

Calderón, L. (2008). Evaluación de la presencia de perros (Canis familiaris) en humedales de la sabana de Bogotá (Colombia) y su efecto potencial sobre la fauna silvestre (Tesis de pregrado). Bogotá: Universidad de los Andes.

del Olmo, C. (2008). Manual de restauración de humedales mediterráneos. Andalucía, España. Junta de Andalucía, Consejería del Medio Ambiente.

Fox, O. 2013. Restauración de humedales urbanos: una oportunidad para la educación ambiental y la participación. Recuperado de: http://www. arquls.cl/2013/05/restauracion-de-humedales-urbanos-una-oportunidad-para-la-educacion-ambiental-y-la-participacion/ 
Gallo, M. y Rodríguez, E. (2010). Caracterización de paisajes y ecosistemas proyecto demostrativo cuenca baja del río Paz El Salvador-Guatemala. Wetlands International. Recuperado de: http://lac.wetlands.org/LinkClick.aspx?fileticket $=8 \mathrm{ddlQPdaYK} 4 \% 3 \mathrm{D} \& \mathrm{tabi}-$ $\mathrm{d}=2138 \& \mathrm{mid}$

García, D. (2013). Humedales del país presentan serios daños, a pesar de la legislación que los protege. Recuperado de: http://www.crhoy.com/archivo/humedales-del-pais-presentan-serios-danos-a-pesar-de-legislacion-que-los-protege/\#.

González, D., Jiménez, J., Jiménez, L. y Palma, M. (2014). Hechos históricos y conflictos del Laguito, además de su influencia hacia los habitantes de San Ramón, Alajuela desde el año 1949 hasta el 2014 (Trabajo de Investigación del curso Seminario de Realidad Nacional II: El Patrimonio Cultural en la Región Occidente). Universidad de Costa Rica, Sede de Occidente, Costa Rica.

Guido, I. y Rodríguez, C. (2011). Avifauna de la ciudad de San Ramón, Alajuela, Costa Rica y su área de expansión urbana. Brenesia 75-76, 23-36.

Horn, S. (2007). Late quaternary lake and swamp sediments: recorders of climate and environmental history. En: J. Bundschuh y G. Alvarado, Central America: geology, resources, hazards, vol 1. (pp. 423-441). Leiden, The Netherlands: Taylor \& Francis/Balkema.

Instituto de Estudios Ambientales/IDEA. (2008). Plan de manejo ambiental del humedal Burro: problemática, valoración y evaluación. Universidad Nacional de Colombia. Recuperado de: http://ambientebogota.gov.co/ documents/10157

Leandro, H., Coto, J., y Salgado, V. (2010). Calidad del agua de los ríos de la microcuenca IV del Río Virilla. Uniciencia, 24, 69-74.
Marín-Muñiz, J.L. y Hernández, M. (2016). Actividades de habitantes de Monte Gordo, Veracruz, como factor en cambios de percepción sobre humedales y fenómenos naturales. RINDERESU, 1(2), 13-23.

Marsik, M. y Waylen, P. (2006). An application of the distributed hydrologic model CASC2D to a tropical montane watershed. Journal of Hydrology, 330, 481-495.

Mora, C. (2007). Nueva propuesta de diseño para el embalse de regulación y uso múltiple "El Laguito", con el fin de prevenir las inundaciones en la ciudad de San Ramón. (Tesis para optar al título de Ingeniero Civil). San José: Universidad de Costa Rica.

Paaby-Hansen, P. (2008). Vacíos en los esfuerzos de conservación de la biodiversidad en aguas continentales de Costa Rica. Recursos Naturales y Ambiente, 54, 28-36.

Quesada, M. (2000). Transformación en el uso de la tierra: un estudio de la microcuenca Quebrada Estero, San Ramón. Revista Geográfica de América Central, 38, 43-59.

Quesada, M. (2001). Evaluación socioambiental de la microcuenca Quebrada Estero, San Ramón Costa Rica. (Informe de investigación). Sede de Occidente de la Universidad de Costa Rica, San Ramón, Costa Rica.

Quesada, M. (2003). Análisis cronológico de los desbordamientos de la Quebrada Estero, San Ramón. Pensamiento Actual, 4(5), 35-41.

Rodríguez, C., Barrantes, T., Guido, I. y González, A. (2012). Proyecto Gestión del Laguito de la Sede de Occidente, UCR. (Propuesta de proyecto de investigación). Universidad de Costa Rica, Sede de Occidente, San Ramón, Costa Rica.

Rodríguez, C. y Silva, M. (2015). Calidad del agua en la microcuenca alta de la Quebrada Estero en San Ramón de Alajuela, Costa Rica. Pensamiento Actual, 15(25), 85-97. 
Rodríguez, C. y Silva, M. (2016). Vegetación acuática en la microcuenca alta de la Quebrada Estero en San Ramón de Alajuela, Costa Rica. Brenesia, 85-86. En prensa.

Sánchez, G., Valle, D., Scorza, F., Feoli, S. y Artavia, R. (2015). Protocolo de Reforestación para la Rehabilitación y Mantenimiento en Áreas de Protección de la GAM. San José, Costa Rica: MINAE, Municipalidad de San José y CNFL.

Secretaría de la Convención de Ramsar. (2010a). Inventario de humedales: Marco de Ramsar para el inventario y la descripción de las características ecológicas de los humedales. (4⿳亠丷a ed.) Secretaría de la Convención de Ramsar, Gland: Suiza

Secretaría de la Convención de Ramsar. (2010b). Manejo de humedales: Marcos para manejar sitios Ramsar y otros humedales. (4⿳亠丷a ed.) Secretaría de la Convención de Ramsar, Gland: Suiza

Secretaría de la Convención de Ramsar. (2013). Manual de la Convención de Ramsar: Guía a la Convención sobre los Humedales (Ramsar, Irán 1971). (6 $6^{\mathrm{a}}$ ed). Secretaría de la Convención de Ramsar, Gland: Suiza
Secretaría Distrital del Ambiente/SDA. (2008). Protocolo de restauración de humedales en entornos urbanos. Bogotá. Recuperado de: http://ambientebogota.gov.co/c/ document_library/get_file?uuid $=88$ baf0e0-bcbe-4df9-86dd-bab29a5740b3\&groupId $=10157$

Sistema Nacional de Áreas de Conservación/ SINAC. (2007). Grúas II. Análisis de vacíos de conservación en Costa Rica: Análisis de Vacios en la Representatividad e Integridad de la Biodiversidad de los sistemas de aguas continentales. San José: SINAC-MINAE.

Sistema Nacional de Áreas de Conservación/ SINAC, Programa de las Naciones Unidas para el Desarrollo/PNUD y Proyecto Humedales. (2015). Documento Base Política Nacional de Humedales Costa Rica y sus Planes de Acción Regionales. Recuperado de: http:// www.politicahumedales.cr/2015/08/documentos.html 


\section{Los Humedales de la Quebrada Estero en San Ramón}

\section{Anexos}

Anexo 1. Lista de chequeo de los servicios ambientales brindados por los ecosistemas acuáticos

\begin{tabular}{|c|c|}
\hline Servicio ecológico & Sí/No \\
\hline Agua potable para seres humanos y/o para el ganado $(\mathrm{P})$ & \\
\hline Agua para la agricultura de regadío $(\mathrm{P})$ & \\
\hline Agua para la industria $(\mathrm{P})$ & \\
\hline Reabastecimiento de aguas subterráneas (R) & \\
\hline Purificación de aguas/tratamiento o dilución de desechos (R) & \\
\hline Alimentos para seres humanos $(\mathrm{P})$ & \\
\hline Alimentos para el ganado $(\mathrm{P})$ & \\
\hline Madera, juncos, fibras y turba $(\mathrm{P})$ & \\
\hline Productos medicinales $(\mathrm{P})$ & \\
\hline Agentes de control biológico para plagas/enfermedades (R) & \\
\hline Otros productos y recursos, incluido material genético $(\mathrm{P})$ & \\
\hline Control de inundaciones, diques de contención de inundaciones (R) & \\
\hline Suelos, sedimentos y retención de nutrientes (R) & \\
\hline Estabilización de la costa y las riberas y protección contra tormentas (R) & \\
\hline Otros servicios hidrológicos (R) & \\
\hline Regulación del clima local / amortiguación del cambio (R) & \\
\hline Almacenamiento/ secuestro del carbono (R) & \\
\hline Caza y pesca con fines recreativos $(\mathrm{C})$ & \\
\hline Deportes acuáticos $(\mathrm{C})$ & \\
\hline Actividades de estudio de la naturaleza (C) & \\
\hline Otras formas de esparcimiento y turismo (C) & \\
\hline Valores educativos $(\mathrm{C})$ & \\
\hline Patrimonio cultural (C), así como los valores de existencia (C) & \\
\hline Valores estéticos y de “sentido de ubicación" (C) & \\
\hline Valores espirituales y religiosos $(\mathrm{C})$ & \\
\hline $\begin{array}{l}\text { Sistemas de conocimientos importantes, e importancia para las investigacio- } \\
\text { nes (C) }\end{array}$ & \\
\hline
\end{tabular}

Fuente. Secretaría de la Convención Ramsar (2010b). 
Anexo 2. Lista de chequeo de las principales amenazas a los humedales y sus impactos

\begin{tabular}{|c|c|c|}
\hline Amenaza & Descripción del impacto & Presencia/ausencia \\
\hline Fragmentación del humedal. & $\begin{array}{l}\text { Pérdida de conectividad ecológica. } \\
\text { Imposibilita el movimiento de algunas especies acuáticas debido a la } \\
\text { construcción de vías, represas, etc. }\end{array}$ & \\
\hline $\begin{array}{l}\text { Deficiente calidad de agua que ingre- } \\
\text { sa al humedal }\end{array}$ & $\begin{array}{l}\text { Eutrofización. } \\
\text { Mala calidad de hábitat para flora y fauna. } \\
\text { Debido a descargas de aguas residuales por ausencia de alcantari- } \\
\text { llado sanitario. }\end{array}$ & \\
\hline $\begin{array}{l}\text { Pérdida acelerada del área inundable } \\
\text { y ronda }\end{array}$ & $\begin{array}{l}\text { Pérdida de área, volumen del cuerpo de agua y del espejo de agua } \\
\text { procesos de urbanización en el área de humedal y por arrastre y } \\
\text { deposición de sedimentos. } \\
\text { Eutrofización. }\end{array}$ & \\
\hline $\begin{array}{l}\text { Pérdida de la capacidad para mitigar } \\
\text { inundaciones }\end{array}$ & $\begin{array}{l}\text { Pérdida de capacidad para atenuar caudales, lo que provoca inun- } \\
\text { daciones y desbordamientos. }\end{array}$ & \\
\hline Déficit hídrico & $\begin{array}{l}\text { Desvío de caudales. } \\
\text { Extracción de agua para otros usos. }\end{array}$ & \\
\hline Depósitos de escombros y basuras & $\begin{array}{l}\text { Tiene efecto sobre la calidad del agua y los suelos del humedal } \\
\text { Inadecuado manejo de desechos sólidos. }\end{array}$ & \\
\hline $\begin{array}{l}\text { Presencia de predadores domésticos } \\
\text { de la fauna }\end{array}$ & $\begin{array}{l}\text { Presencia de ratas, perros y gatos que atacan la escasa fauna que } \\
\text { aún persiste en el humedal. }\end{array}$ & \\
\hline Pastoreo de animales & $\begin{array}{l}\text { Compactación del suelo y aportes de excrementos al cuerpo de } \\
\text { agua. }\end{array}$ & \\
\hline $\begin{array}{l}\text { Modificación en la composición y es- } \\
\text { tructura de las comunidades bióticas }\end{array}$ & $\begin{array}{l}\text { Pérdida de especies nativas } \\
\text { proliferación de especies invasivas } \\
\text { Pérdida de especies acuáticas susceptibles a las altas cargas orgáni- } \\
\text { cas del agua y a la baja oxigenación. }\end{array}$ & \\
\hline $\begin{array}{l}\text { Apropiación y uso inadecuado de las } \\
\text { áreas de ronda }\end{array}$ & $\begin{array}{l}\text { Apropiación de estas áreas para desarrollar proyectos de construc- } \\
\text { ción de vivienda. }\end{array}$ & \\
\hline Actividad urbana adyacente & $\begin{array}{l}\text { Impactos por el tráfico, barreras a la movilidad, inseguridad, con- } \\
\text { taminación con basuras y vertimientos, etc., incompatibilidades de } \\
\text { uso del suelo. }\end{array}$ & \\
\hline Obras de infraestructura vial & $\begin{array}{l}\text { Se generan tanto en la etapa de construcción, como en las etapas de } \\
\text { operación de las vías produciendo ruido, contaminación atmosféri- } \\
\text { ca y alteraciones del patrón de flujo hídrico entre otros. }\end{array}$ & \\
\hline $\begin{array}{l}\text { La ausencia de una valoración am- } \\
\text { biental y económica de los servicios } \\
\text { ecosistémicos que provee el humedal, } \\
\text { desconocimiento de estos por el grue- } \\
\text { so de la sociedad. }\end{array}$ & $\begin{array}{l}\text { Facilita el deterioro y la sobreexplotación, ya que a la hora de tomar } \\
\text { decisiones sobre el uso que se le debe dar a estos ecosistemas los } \\
\text { criterios económicos generalmente priman por encima de conside- } \\
\text { raciones ecológicas o éticas. }\end{array}$ & \\
\hline $\begin{array}{l}\text { La falta de control y vigilancia en el } \\
\text { humedal }\end{array}$ & $\begin{array}{l}\text { Las autoridades competentes no realizan un adecuado control y } \\
\text { vigilancia, lo que propicia el depósito de basuras y el desarrollo de } \\
\text { actividades inadecuadas en el humedal. }\end{array}$ & \\
\hline $\begin{array}{l}\text { Problemas relacionados con los dere- } \\
\text { chos de propiedad y uso }\end{array}$ & $\begin{array}{l}\text { Existencia de predios privados en la zona de humedal y zona de } \\
\text { ronda. }\end{array}$ & \\
\hline $\begin{array}{l}\text { Desarticulación de las actividades de } \\
\text { las instituciones pertinentes }\end{array}$ & $\begin{array}{l}\text { Esfuerzos aislados e incompletos, escasa protección, ausencia de } \\
\text { manejo, etc. }\end{array}$ & \\
\hline Uso del suelo & $\begin{array}{l}\text { Puede provocar contaminación, erosión, aumento de la escorrentía, } \\
\text { etc. }\end{array}$ & \\
\hline
\end{tabular}

Fuente. IDEA (2008) 